\title{
WWOX-mediated apoptosis in A549 cells mainly involves the mitochondrial pathway
}

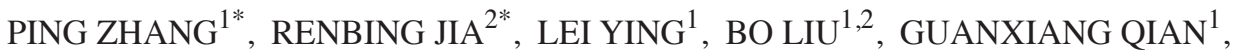 \\ XIANQUN FAN ${ }^{2}$ and SHENGFANG GE ${ }^{1,2}$ \\ ${ }^{1}$ Department of Biochemistry and Molecular Biology, Shanghai Jiaotong University School of Medicine; \\ ${ }^{2}$ Department of Ophthalmology, Shanghai Ninth People's Hospital Affiliated to Shanghai \\ Jiaotong University School of Medicine, Shanghai, P.R. China
}

Received December 21, 2011; Accepted March 20, 2012

DOI: $10.3892 / \mathrm{mmr} .2012 .860$

\begin{abstract}
The human WWOX gene, known as WW domaincontaining oxidoreductase, is located on 16q23.3-24.1, a chromosome region that spans the common fragile site, FRA16D. Abnormal transcripts or even loss of expression are frequently found in a number of cancer cell types, including breast, ovarian, prostate and lung cancer cells. It has therefore been proposed that the WWOX gene encodes a candidate tumor suppressor, possibly a pro-apoptotic protein. However, the mechanism behind this is not entirely clear. In the present study, we examined the pro-apoptotic action of WWOX using transient expression in A549 cells. We observed that the ectopic expression of WWOX caused apoptosis in A549 cells. We further observed procaspase-3 and procaspase- 9 activation and the release of cytochrome $C$ from the mitochondria in A549 cells transfected with pcDNA3.0-WWOX. These data indicate that WWOX induces apoptosis in A549 cells via the mitochondrial pathway.
\end{abstract}

\section{Introduction}

Human WW domain-containing oxidoreductase (WWOX) is located on 16q23.3-24.1, a chromosome region that spans the common fragile site, FRA16D (1). The wild-type WWOX gene encodes a $46-\mathrm{kDa}$ protein, containing $2 \mathrm{~N}$-terminal WW domains, a nuclear localization sequence (NLS) and a C-terminal short-chain alcohol dehydrogenase/reductase

Correspondence to: Professor Xianqun Fan or Professor Shengfang Ge, Department of Ophthalmology, Shanghai Ninth People's Hospital Affiliated to Shanghai Jiaotong University School of Medicine, 639 Zhi Zao Ju Road, Shanghai 200011, P.R. China

E-mail: fanxq@sh163.net

E-mail: geshengfang@hotmail.com

*Contributed equally

Key words: WW domain-containing oxidoreductase, mitochondrion, apoptosis
(ADH/SDR) domain (1). The human WWOX gene has 9 exons. To date, seven alternatively spliced WWOX protein variants have been identified. The aberrant expression of WWOX has been reported in various cancer cell types including breast (2), ovarian (3), prostate (4), gastric (5) and hepatic cancer (6), osteosarcoma (7), and lung cancer (8).

Nunez et al reported that in the tissue of 38 human ovarian carcinomas, one third of the cases showed extremely reduced or absent WWOX protein expression (9). Aqeilan et al analyzed a set of 55 patient samples with gastric adenocarcinoma using immunohistochemical staining. Their results revealed that $65 \%$ of the samples were negative for the WWOX protein (5). Yendamuri et al also examined the WWOX protein expression pattern in non-small cell lung cancer. Their studies revealed that transcripts lacking WWOX exons were detected in 7 primary tumors ( 7 of $27 ; 25.9 \%$ ) and loss of heterozygosity at the WWOX locus was observed in 10 primary tumors (10 of $27 ; 37.0 \%$ ) (10). Due to the low or even undetectable level of wild-type WWOX gene expression and frequent appearance of variants in these tumor cells, the WWOX protein is considered to be a tumor suppressor.

Two classic pathways for the induction of apoptosis have been identified to date: the first of these is elicited by a death receptor in the plasma membrane (extrinsic pathway) and the second involves mitochondria (intrinsic pathway). The mechanism by which WWOX induces apoptosis is far from clear. Analysis has produced the hypothesis that WWOX has no N-terminal signal peptide (1); therefore, its localization is possibly in the cytoplasm. In addition, the mitochondrial targeting sequence is mapped within the $\mathrm{ADH}$ domain (11). The Tyr33 in the first WW domain sequence is the key amino acid that can be phosphorylated (12). Phosphorylated WWOX can be considered to be an activated form, which is essential for its migration to the mitochondria from the cytoplasm (12). Apoptotic stress results in the release of 'activated' WWOX from the mitochondria and subsequent nuclear translocation (13).

From these data, we speculated that WWOX performs a pro-apoptotic role in the mitochondria. In this study, to investigate the mechanisms by which WWOX induces apoptosis, we focused on those data concerning the mitochondrial apoptotic pathway. The pro-apoptotic action of WWOX was examined 
using transient expression in A549 cells. Our results revealed the release of cytochrome $C$ and the activation of caspase-3 and caspase-9.

\section{Materials and methods}

Cell lines and culture. The A549 human lung adenocarcinoma cell line [American Type Culture Collection (ATCC)], was grown in monolayer culture at $37^{\circ} \mathrm{C}$ and $5 \% \mathrm{CO}_{2}$ in Dulbecco's modified Eagle's medium (DMEM) (Gibco BRL, Gaithersburg, MD, USA) supplemented with $10 \%$ fetal calf serum (HyClone, Waltham, MA, USA), $100 \mathrm{U} / \mathrm{ml}$ of penicillin (Gibco) and $100 \mu \mathrm{g} / \mathrm{ml}$ of streptomycin (Gibco).

Plasmid constructs and transient transfections. Full-length WWOX cDNA was obtained from human fetal spleen total RNA by RT-PCR, using the following primers: forward, 5'-ACGAAGCTTTTGGAGCGGGAGTGAGT-3'; and reverse, 5'-AGCGGATCCGTTGGAGGGACATTTGG-3'. HindIII and BamHI sites are shown in bold. The PCR conditions were as follows: $95^{\circ} \mathrm{C}$ for $3 \mathrm{~min}, 30$ cycles at $94^{\circ} \mathrm{C}$ for $1 \mathrm{~min}, 56^{\circ} \mathrm{C}$ for $1 \mathrm{~min}, 72^{\circ} \mathrm{C}$ for $1.5 \mathrm{~min}$ and a further $72^{\circ} \mathrm{C}$ for $10 \mathrm{~min}$. The mammalian expression plasmid, pcDNA3.0WWOX, was constructed by ligating the WWOX PCR products and the pcDNA3.0 vector, which were digested with HindIII and BamHI. pcDNA3.0 was used as the control plasmid. Overexpression of the proteins was achieved by transient transfection with Lipofectamine 2000 (Invitrogen, Carlsbad, CA, USA) reagent according to the manufacturer's instructions.

Cell proliferation. Cell proliferation was determined using the methyl thiazolyl tetrazolium (MTT) assay. A549 cells (1x104) were planted in 96-well plates $24 \mathrm{~h}$ prior to transfection. At 24,48 and $72 \mathrm{~h}$ post-transfection with pcDNA3.0-WWOX or the control plasmid pcDNA3.0, the MTT reagent was added to each well. After $4 \mathrm{~h}$ of incubation at $37^{\circ} \mathrm{C}$, DMSO was used to dissolve the resulting formazan crystals and absorbance was recorded at $540 \mathrm{~nm}$ in a microplate reader (Sunrise).

Apoptosis assay. For cell apoptosis analysis, A549 cells $\left(1 \times 10^{6}\right)$ in 6-well plates were harvested $24 \mathrm{~h}$ post-transfection. The cell pellets were washed twice with cold PBS buffer, then re-suspended in $1 \mathrm{ml}$ Annexin V binding buffer. The solution mentioned above $(100 \mu \mathrm{l})$ was transfered to another culture tube and incubated with $5 \mu \mathrm{l}$ Annexin V-FITC and $5 \mu \mathrm{l}$ propdium iodide (PI) (BD Pharmingen, San Diego, CA, USA) for $15 \mathrm{~min}$ at room temperature in the dark. The cell suspension was diluted with $400 \mu \mathrm{l}$ PBS buffer and $1 \times 10^{5}$ cells were assayed in FACSCalibur (BD Biosciences) as soon as possible.

Western blot analysis. Total proteins were extracted from pcDNA3.0 or pcDNA3.0-WWOX-transiently transfected A549 cells in a 6-cm dish for pro-caspase activation detection. The cytosolic proteins were obtained using differential centrifugation. Briefly, the cells were suspended in a buffer containing $0.25 \mathrm{M}$ sucose, $50 \mathrm{mM}$ HEPES, $0.1 \mathrm{mM}$ EDTA (pH 7.4), $10 \mathrm{mM} \mathrm{NaCl}, 2 \mathrm{mM}$ DTT and protease inhibitor cocktail (Sigma, St. Louis, MO, USA) on ice for $10 \mathrm{~min}$, homogenized by 40 passes in an ice-cold Dounce grinder and centrifuged at $700 \mathrm{x} \mathrm{g}$ for $10 \mathrm{~min}$. After removing the debris, the resulting supernatant was further centrifuged at 10,000 $\mathrm{x} g$ for $30 \mathrm{~min}$ at $4^{\circ} \mathrm{C}$. The supernatants were collected as cytosolic fractions. These proteins were identified using a BCA protein assay kit (Pierce, Rockford, IL, USA). Protein $(20 \mu \mathrm{g})$ was resolved by $15 \%$ SDS-PAGE and transferred to PVDF membranes (Millipore, Billerica, MA, USA). The membrane was blocked with blocking buffer [5\% non-fat dry milk in $20 \mathrm{mM}$ Trisbuffered saline ( $\mathrm{pH} 7.5$ ) with $0.1 \%$ Tween-20] for $2 \mathrm{~h}$ at room temperature followed by incubation with appropriate primary antibodies at the dilutions of 1:2000 overnight at $4{ }^{\circ} \mathrm{C}$. The membrane was then incubated with appropriate secondary antibody for $2 \mathrm{~h}$ and examined using the enhanced chemiluminescence western blotting detection kit (Pierce). Rabbit polyclonal antibody to human WWOX was purchased from Chemicon International Inc. (Billerica, MA, USA). Rabbit polyclonal antibodies to human caspase-9, caspase-3 and cytochrome $C$ were purchased from Cell Signaling Technology (Beverly, MA, USA). $\beta$-tubulin was purchased from Santa Cruz Biotechnology (Santa Cruz, CA, USA).

\section{Results}

WWOX inhibits cell proliferation. The effects of WWOX protein expression on the proliferation of A549 cells were measured by MTT assay. A significant anti-proliferative effect was observed in the cells exposed to pcDNA3.0-WWOX transfection. At 24, 48 and $72 \mathrm{~h}$, the results showed that the WWOX protein reduced cell proliferation. The cell proliferative percentage decreased to $85.62 \pm 3.27,75.47 \pm 3.15$ and $73.04 \pm 1.79 \%$ at 24,48 and 72 h, respectively (Fig. 1). Data are presented as the means \pm SEM.

WWOX induces cell apoptosis. To investigate whether the WWOX-mediated apoptosis resulted in the decreased proliferative ratio of A549 cells, we examined the early apoptotic cells using Annexin V-FITC/PI staining analysis $24 \mathrm{~h}$ post-transfection. Cells transfected with pcDNA3.0-WWOX exhibited an early apoptotic rate of $16.64 \pm 3.04 \%$ represented in the low-right quadrant, which was significantly higher than the $3.0 \pm 0.48 \%$ observed in the control cells (Fig. 2). Data are presented as the means \pm SEM.

WWOX triggers the release of cytochrome $C$ from mitochondria. The release of cytochrome $C$ from the mitochondria to cytosol was measured by western blot analysis as described above. Cells transfected with pcDNA3.0-WWOX or control plasmid were assayed at $24 \mathrm{~h}$ post-transfection. The analysis revealed a marked elevation in the level of cytosolic cytochrome $C$ of the cells transfected with pcDNA3.0-WWOX compared to that of the control cells (Fig. 3).

WWOX activates caspase-9 and caspase-3. The activation of caspase-9 and caspase-3 was involved in the mitochondrionmediated apoptotic pathway. In the pcDNA3.0-WWOX transfected cells, we observed the full-length caspase-9 accompanied by an increase in the 35- and 37-kDa cleaved protein bands representing a subunit of activated caspase-9. A parallel increase of a $17-\mathrm{kDa}$ cleaved protein band was also observed in caspase-3 activation analysis (Fig. 4). 


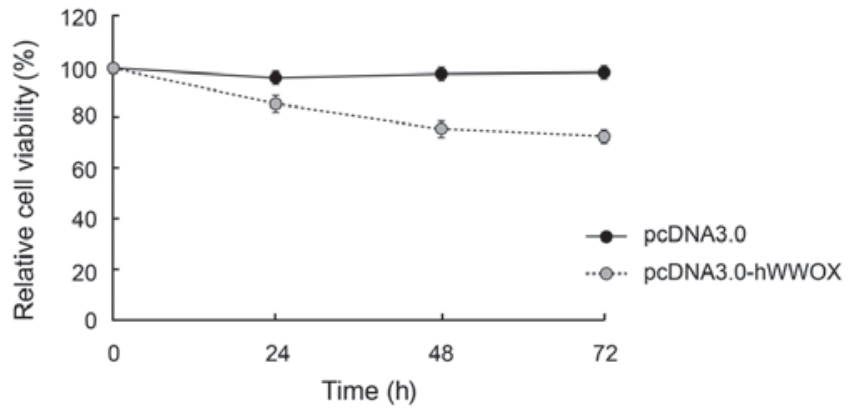

Figure 1. Effect of WWOX on the cell proliferation of A549 cells. MTT assay was performed after 24,48 and $72 \mathrm{~h}$ of transfection. Cellular viability was determined as an absorbance at $570 \mathrm{~nm}$. The cell proliferative percentage decreased to $85.62 \pm 3.27 \%, 75.47 \pm 3.15 \%$ and $73.04 \pm 1.79 \%$ at 24,48 and $72 \mathrm{~h}$, respectively. Data are presented as the means \pm SEM.

A

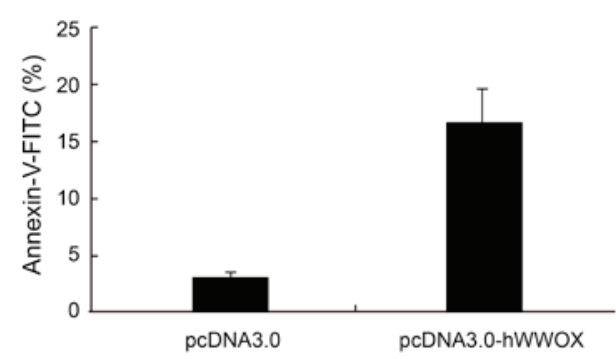

B
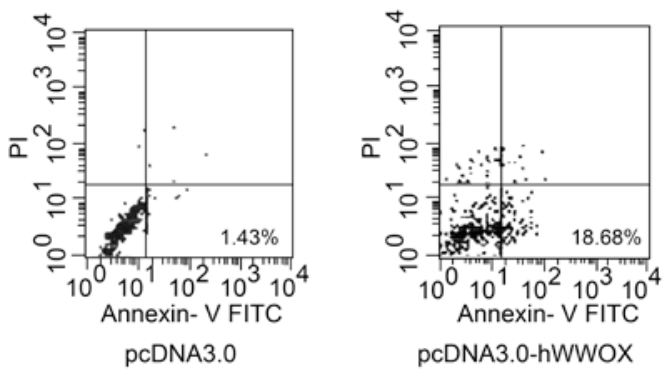

Figure 2. Induction of apoptosis in the A549 cell line by WWOX. Flow cytometry analysis was used to examine the apoptotic cells $24 \mathrm{~h}$ post-transfection. (A) Cells transfected with pcDNA3.0-WWOX had an early apoptotic rate of $16.64 \pm 3.04 \%$, whereas the control cells had an early apoptotic rate of only $3.0 \pm 0.48 \%$. Data are presented as the means \pm SEM. (B) Representative FCA graph of cells at early apoptosis (Annexin-V-positive and PI-negative).

\section{Discussion}

In this study, we provide evidence that the mitochondrial apoptotic pathway is activated in A549 cells in the WWOXmediated apoptosis. In our experiments, we examined the pro-apoptotic action of WWOX using transient expression in A549 cells. When expressed, WWOX induced cell apoptosis in conjunction with pro-caspase- 3 and pro-caspase- 9 activation and cytochrome $C$ release from the mitochondria. These data indicate that WWOX induces apoptosis via the mitochondrial pathway.

The WWOX protein has several functional domains: 2 N-terminal WW domains and a C-terminal alcohol dehydrogenase domain (ADH domain). The enzymatic function of the ADH domain is related to oxidation/reduction of lipid hormones and metabolic mediators, such as androgens and

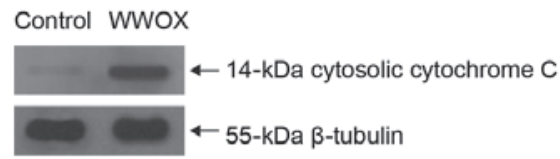

Figure 3. Release of cytochrome $C$ from the mitochondrion to the cytosol triggered by WWOX. Cells transfected with pcDNA3.0-WWOX or control plasmid were homogenized in proper buffer $24 \mathrm{~h}$ post-transfection respectively, and the same amount of cytosolic fraction was examined for the released cytochrome $C$. Western blot analysis showed increased cytochrome $C$ expression in the cytosol in pcDNA3.0-WWOX-transfected cells compared to the control cells. $\beta$-tubulin was used as the internal control.
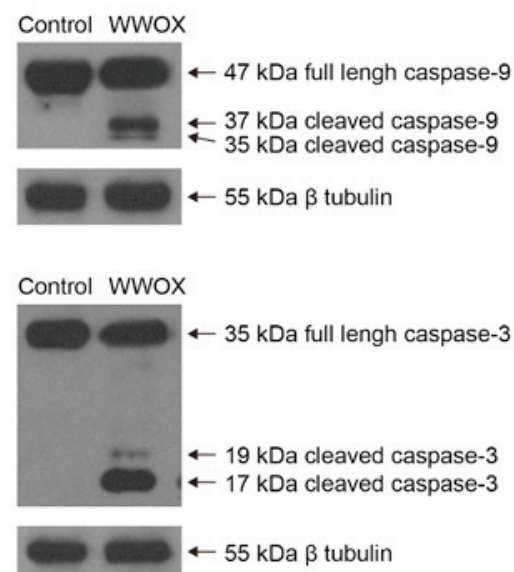

Figure 4. Activation of caspase- 9 and caspase- 3 by WWOX. Cells transfected with pcDNA3.0-WWOX or control plasmid were lysed $24 \mathrm{~h}$ post-transfection and the same amount of lysate was examined for the activated form of caspase- 9 and caspase- 3 by western blot analysis. The full-length caspase- 9 was $47-\mathrm{kDa}$. We detected the 35- and 37-kDa bands representing the cleaved fragment of caspase-9 in the cells transfected with pcDNA3.0-WWOX. The full-length caspase-3 was $35-\mathrm{kDa}$. The 17 - and $19-\mathrm{kDa}$ bands indicating the cleaved fragment of caspase- 3 were detected in the cells transfected with pcDNA3.0-WWOX. None of these cleaved fragments were observed in the control cells. $\beta$-tubulin was used as the internal control.

estrogens (14). This corresponds to the abundance of WWOX mRNA in hormone-regulated tissues, including breast, ovary, testis and prostate tissue. However, as regards the induction of cell apoptosis, the function of the ADH domain is considered to be as a location effector in the mitochondria, based on a mitochondrial targeting sequence within the $\mathrm{ADH}$ domain (11). Chang et al examined endogenous WWOX localization by antibodies against various regions in WWOX and fluorescent fusion protein technology and confirmed that WWOX is clustered in the mitochondria (12). We therefore speculated that WWOX translocates to the mitochondrion in response to apoptotic stress, affecting the mitochondrial intermembrane stability. When the mitochondrial intermembrane pores open, procaspase-3, procaspase-9, cytochrome $C$ and apoptosis-inducing factor are released from the mitochondrial intermembrane space to the cytoplasm. This may initiate/ trigger the mitochondrial apoptotic pathway.

p53 is a well-known apoptosis-inducing protein. The WW domains in WWOX have the function of interaction with the proline-rich motif in p53. The proline-rich motif is the essential region for p53-mediated apoptosis (15). Chang et al determined 
that WWOX is the key factor of p53 in apoptosis (11). WWOX may therefore enhance the apoptosis-inducing activity of p53 (6). In addition, A549 is a p53-positive cell line. Previous studies have shown that exogenous WWOX clearly induces A549 cell apoptosis (16). The induction of A549 cell apoptosis by WWOX is therefore partly involved in the enhancement of the apoptosis-inducing ability of p53. There are 2 Tyr (Tyr33 and Tyr61) in the first WW domain, which can be phosphorylated. The phosphorylation of these 2 Tyr is considered to be the key step in mediating migration to the mitochondria. Chang et al also observed the elimination of apoptosis induced by WWOX with replacement of the Tyr33 to Arg33 (17). Moreover, they observed the phosphorylation of WWOX with a specific antibody against phosphor-Tyr33 peptide corresponding to the first WW-domain sequence in WWOX (11).

In conclusion, our data show that WWOX is involved in cell apoptosis, mainly through the mitochondrial pathway. Understanding the mechanism of WWOX-induced apoptosis may provide novel targets in cancer gene therapy.

\section{Acknowledgements}

This study was supported by the Natural Science Foundation of China (31000662), the Elevation Project for Young Teachers from the Shanghai Jiaotong University School of Medicine, the Shanghai Leading Academic Discipline Project (S30205), and the Shanghai Rising-Star Program (11QA1404000).

\section{References}

1. Bednarek AK, Laflin KJ, Daniel RL, Liao Q, Hawkins KA and Aldaz CM: WWOX, a novel WW domain-containing protein mapping to human chromesome 16q23.3-24.1, a region frequentlly affected in breast cancer. Cancer Res: 60: 2140-2145, 2000.

2. Pluciennik E, Kusiñska R, Potemski P, Kubiak R, Kordek R and Bednarek AK: WWOX-the FRA16D cancer gene: expression correlation with breast cancer progression and prognosis. Eur J Surg Oncol 32: 153-157, 2006.

3. Paige AJ, Zucknick M, Janczar S, Paul J, Mein CA, Taylor KJ, Stewart M, Gourley C, Richardson S, Perren T, et al: WWOX tumour suppressor gene polymorphisms and ovarian cancer pathology and prognosis. Eur J Cancer 46: 818-825, 2010.

4. Cheng I, Levin AM, Tai YC, Plummer S, Chen GK, Neslund-Dudas C, Casey G, Rybicki BA and Witte JS: Copy number alterations in prostate tumors and disease aggressiveness. Genes Chromosomes Cancer 51: 66-76, 2012.
5. Aqeilan RI, Kuroki T, Pekarsky Y, Albagha O, Trapasso F, Baffa R, Huebner K, Edmonds P and Croce CM: Loss of WWOX expression in gastric carcinoma.Clin Cancer Res 10: 3053-3058, 2004.

6. Aderca I, Moser CD, Veerasamy M, Bani-Hani AH, BonillaGuerrero R, Ahmed K, Shire A, Cazanave SC, Montoya DP, Mettler TA, et al: The JNK inhibitor SP600129 enhances apoptosis of HCC cells induced by the tumor suppressor WWOX. J Hepatol 49: 373-383, 2008.

7. Kurek KC, Del Mare S, Salah Z, Abdeen S, Sadiq H, Lee SH, Gaudio E, Zanesi N, Jones KB, DeYoung B, et al: Frequent attenuation of the WWOX tumor suppressor in osteosarcoma is associated with increased tumorigenicity and aberrant RUNX2 expression. Cancer Res. 70:5577-5586, 2010.

8. Becker S, Markova B, Wiewrodt R, Hoffarth S, Hähnel PS, Pleiner S, Schmidt LH, Breitenbuecher F and Schuler M: Functional and clinical characterization of the putative tumor suppressor WWOX in non-small cell lung cancer. J Thorac Oncol 6: 1976-1983, 2011.

9. Nunez MI, Rosen DG, Ludes-Meyers JH, Abba MC, Kil H, Page R, Klein-Szanto AJ, Godwin AK, Liu J, Mills GB et al: WWOX protein expression varies among ovarian carcinoma histotypes and correlates with less favorable outcome.BMC Cancer 5: 64, 2005.

10. Yendamuri S, Kuroki T, Trapasso F, Henry AC, Dumon KR, Huebner K, Williams NN, Kaiser LR and Croce CM: WW domain containing oxidreductase gene expression is altered in non-small cell lung cancer. Cancer Res 63: 878-881,2003.

11. Chang NS, Pratt N, Heath J, Schultz L, Sleve D, Carey GB and Zevotek N: Hyaluronidase induction of a WW domain-containing oxidoreductase that enhances tumor necrosis factor cytotoxicity. J Biol Chem 276: 3361-3370, 2001.

12. Chang NS, Doherty J, Ensign A, Lewis J, Heath J, Schultz L, Chen ST and Oppermann U: Molecular mechanism underlying WOX1 activation during apoptotic and stress responses. Biochem Pharmacol 66: 1347-1354, 2003.

13. Chang NS:A potential role for p53 and WOX1 in mitochondrial apoptosis. Int J Mol Med 9: 19-24, 2002.

14. Oppermann UC, Filling $\mathrm{C}$ and Jornvall $\mathrm{H}$ : Forms and functions of human SDR enzymes. Chem Biol Interact 30: 669-705, 2001.

15. Zhu J, Zhang S, Jiang J and Chen X: Definition of the p53 functional domains necessary for inducing apoptosis. J Biol Chem 275: 39927-39934, 2000.

16. Zhang P, Ying L, Xu R, Ge S, Mei W, Li F, Dai B, Lu J and Qian G: Tumor-specific, hypoxia-regulated, WW domain-containing oxidoreductase-expressing adenovirus inhibits human non-small cell lung cancer growth in vivo. Hum Gene Ther 21: 27-39, 2010.

17. Chang NS, Doherty J and Ensign A: JNK1 physically interacts with WW domain-containing oxidoreductase(WOX1) and inhibits WOX1-mediated apoptosis. J Biol Chem 278: 9195-9202, 2003. 